\title{
Opinion
}

\section{Horticultural Therapy: An Ancient Technique in a Modern World}

\author{
Gary $_{\text {Altman }}{ }^{*}$ and Nrupali Patel ${ }^{1}$ \\ ${ }^{1}$ Department of Plant Biology, Rutgers University, New Jersey, USA \\ *Corresponding author email: gla32@sebs.rutgers.edu \\ https://doi.org/10.36018/dsiij.v17i.208
}

Gary Altman, MS, CRC, HTR, Teaching Instructor, Associate Director of the Horticultural Therapy Program

Nrupali Patel, Ph.D.Plant Biology Undergraduate Program Director, Teaching Instructor, Research Plant Microbiologist

\begin{abstract}
Horticultural therapy utilizes plants and plant-based activities in the treatment and rehabilitation of individuals with various disabilities. The practice has been in use since the time of ancient Egypt, Hindus and other cultures. In the United States, The American Horticultural Therapy Association is the professional society that has established educational and training standards for those wishing to practice in the profession of horticultural therapy. Various studies have shown the positive mental and physiological healing benefits of plantbased activities. While horticultural therapy has not achieved the evidence-based practice standard on its own, this treatment approach is flexible and adaptable enough to be integrated into existing scientific evidence-based therapy practices in mental healthcare. Despite current challenges with horticultural therapy practice, the use of plants and plant-based activity as a therapeutic method will continue to have a role in the healthcare community. Plants and gardening activity are benign and non-threatening, making their use in therapeutic treatments an appealing approach to populations worldwide.
\end{abstract}

Keywords. Horticulture Therapy, Plants, Healthcare, Cognitive behavioral interventions, Vocational rehabilitation interventions

\section{Introduction and Brief History}

Horticultural therapy is a treatment practice that utilizes plants and plant-based activities in the treatment and rehabilitation of individuals with various disabilities (1). The practice has been in use since the time of ancient Egypt. In horticultural therapy, the use of plants is often a well-received treatment method since horticulture, plants, and gardening activities have cultural significance in many diverse populations. Various cultures such as the Hindu culture recognize the connection with nature and plants as a form of healing,as reflected in Naturopathy and Ayurveda.Horticultural therapists often seek to utilize this connection as part of the treatment or rehabilitation process. For many populations, plants are deeply engrained in the folklore, culture, food, and medicinal practices. In 
many cultures, plants have become recognized as having divine qualities for which they are used in religious practices (2). Simply put, plants have a multicultural appeal that lends itself well to a healthcare treatment. In the western world, however, the "roots" of horticultural therapy began as fieldwork-based treatment for individuals with psychiatric conditions. This was a common practice until horticulture was formalized into a treatment method in the 1800s when the Asylum for Persons Deprived of their Reason (now known as Friends Hospital) opened in Philadelphia, PA (3). This was the first private psychiatric institution in the United States and is considered the birthplace of horticultural therapy, innovating treatment of psychiatric conditions by utilizing the calming effects of the natural environment as a viable treatment tool (3).

Over time, the practice of using horticultural therapy was extended to be used as not only a treatment for individuals with psychiatric conditions, but also as a rehabilitative method for physical conditions afflicting veteran soldiers after World War I and II (3). At this point in time, horticultural therapy was practiced by occupational therapy professionals throughout the 1960's (3). Beginning in the late 1960's and early 1970's, practitioners of horticultural therapy began to recognize a need to formally organize and develop the practice of horticultural therapy into its own unique profession separate from occupational therapy (3). With this, the professional society now known as the American Horticultural Therapy Association (AHTA) was formed. AHTA has established educational and training standards for horticultural therapy professionals (4). The formation of the AHTA has had a significant role in the current practice of horticultural therapy in the US as we know it today.

\section{The rational and theories behind Horticulture Therapy}

The aim for horticultural therapists is to utilize the human psychological and physiological responses of exposure to plants to maximize treatment and rehabilitation outcomes of patients. To be successful in utilizing plants in this healing application, horticultural therapists must be competent in knowledge of plant growth, structure, and function, as well as various theories and applications of psychology and human development. The theoretical basis for the use of plants in horticultural therapy is based upon three theories (5):

1. Biophilia hypothesis, which proposes that humans have an innate relationship with nature that is linked to our biology and psychology.

2. Attention restoration theory, which proposes that the natural environment has a restorative effect on humans.

3. Psychoevolutionary theory of stress reduction, which proposes that the visual properties of natural environments evoke physiological responses associated with calmness, relaxation, and fascination.

\section{The practice, research, and future of horticultural therapy}

The practice of horticultural therapy includes a broad array of services that can be implemented in a variety of practice settings to various populations. The practice can be divided into three areas of treatment programs: vocational, therapeutic, and social. Each of these are differentiated by their goals and expected outcomes for program participants (6). Horticultural therapy provided through vocational programs has a focus on job skills training, job placement and supported employment, while the therapeutic programs are offered in clinical settings (i.e., hospitals, and rehabilitation facilities) and have 
a focus on symptom remediation and/or recovery from illness and injury (6). The social model of horticultural therapy differs from the other models, as theseprogramsfocus on goals of life enrichment and personal development of individuals (6). All three program models are described as having much in common, as all the models aim to maximize health, independence, and self-actualization of the patient (6). The models are also aligned with the practices and aims of the broader area of study of psychiatric rehabilitation, which seek to influence strengths and challenges related to client goals in the community context in which they will be carried out (that is, working in the community, developing skills to socialize with friends and family, improving physical stamina in the garden to improve independence in the home, etc.) (7).

Current research continues to demonstrate the positive psychological and physiological healing benefits of plant-based activity. In a recent study, it was shown that community gardens (nature-based activities) were valid and supportive for social, mental, and physical health of refugees (8). Several emerging themes developed from the subjects, such as improved social connections with friends, family and the community. The gardening process created an improved sense of "community" and also improved food security, as gardening is a sustainable food source that can increase a sense of well-being through the presence of positive emotions while gardening within a community context (8). Sia and Diehl (2020) (9), presented a case study in Singapore that also demonstrated the positive effects of nature-based activities for older adults. As a result of the therapeutic horticulture activities, participants indicated a positive shift in their before and after session happiness levels using the simple pre- and post-test visual analogue scale (10). The concluding data suggested that there were significant improvements and positive effects in participant outlook and optimism.

The mental health in individualswho have served in the military is of great concern to many nations. Recent studies have examined the psychological benefits of horticultural therapy for this population. In the United States, university veteran students often experience challenges integrating from active military duty back into the civilian lifestyle, which affects the individual's interpersonal relationships, ability to obtain and maintain employment, and alsopursue education by "going back to school”(11). A study was conducted to determine the effects of specific greenhouse activities on depression, anxiety, and stress levels of students who served in the U.S. Armed Forces. The data revealed that participation in a plant care class had decreased the levels of depression and stress in students when compared with the control group. Student veterans also described anobvious feeling of reduced stress and the ability to relax with a sense of belonging (11). The results of this study suggest that regular exposure to plants and horticulture can provide an avenue for veterans to address many psychological issues that have the potential to impede readjustment to civilian life.

While there is much qualitative data on the effects of horticultural therapy in various populations indicating positive effects on lifestyle and overall wellness, there is limited quantitative research and data on the effectiveness of plant-based activities that provide a definitive lead to a useful protocol of methodology. Building upon this concept of evidence-based research (12), used near-infrared spectroscopy (NIRS) research to investigate the activation of the pre-frontal areas of the brain in 
healthy older adults while they repeatedly performed plant-based activities. The results indicate that these plant-based activities stimulate the frontal lobe of the brain and potentially contribute to the maintenance for cognitive functions.

Horticultural therapy is often viewed as a therapeutic practice in rehabilitation science, occupational therapy, or as a form of recreational therapy, however, an argument can be made for horticultural therapy to be considered as a creative arts therapy method that is akin to the more well-known creative arts therapies such as art, music, and dance/movement therapy. The creative arts therapies are typically defined as art forms that are primarily auditory or written (i.e., music, drama, and literature) or visual (i.e., painting, dance) that are combined with therapeutic skills in a counseling context (13). Horticulture is commonly thought of as a set of technical skills, however, horticulture is both a science as well as an art. While it is true that many plants may grow and survive if simply just planted, horticulture involves care and training, and requires a level of commitment, imagination, measured risks, and appreciation much like the other more wellknown creative arts therapies (14). Like all creative arts therapies, horticulture involves an element of unpredictability, specifically how or if a plant will grow and develop, and this unpredictability is a common link between the use of horticulture as a therapeutic methodsince this unpredictability leads to opportunities for therapeutic interaction between the therapist and patient (15). The unpredictability of nature and how an individual relates to it provides an opportunity for patients in treatment to learn the nature of themselves and the world around them and has the potential to affect the patient's world view, and how they understand themselves in the context of the world or community. This change in world view relates to spirituality in the sense it introduces therapeutic concepts such as recovery, hope, innerpeace, and well-being.

There has been a long history of using plants and plant-based activity in treatment and it has taken more than 40 years for the profession to reach the current status of development. Presently, horticultural therapists have developed models of practice that have been widely accepted by the profession (i.e., therapeutic, vocational, and social models), however, these models are broad reaching and not well defined in terms of describing when and under what conditions should the model be applied. This ambiguity, and a lack of detailed horticultural therapy program descriptions have created a tendency for horticultural therapists to rely on clinical decision-making based upon knowledge gathered through practice, rather than scientific research (16). As a result, there is broadscalevariability in the quality and intensity of horticultural therapy services provided to individuals who are recovering from illness or injury, or adjusting to disability.

The current trends in the U.S. healthcare are moving towards the development of evidence-based practices, as research has demonstrated that evidence-based practices can improve the quality of care provided and overall outcomes of treatment (17). Evidence-based practices are not a new concept in mental health care, as there are numerous models that have been developed to address various psychiatric rehabilitation objectives. There is some evidence that horticultural therapy and other creative arts therapies canaid in psychiatric health care when offered in conjunction to evidence-based practices, by enhancing self-knowledge, awareness, and improved relationship skills (7). While 
horticultural therapy has not achieved the evidencebased practice standard on its own, the practice is flexible and adaptable enough to be integrated with existing evidence-based practices within mental healthcare.

It is without question that individuals with serious mental illness should receive care based upon the best evidence available. Regarding horticultural therapy, however, should the practice continue as an adjunctivetherapy intervention, or should the professionadopt an evidence-based practice approach to work with patients?For the practice of horticultural therapy to continue to gain traction as an acceptable healthcare treatment in the U.S. and worldwide in the $21^{\text {st }}$ century, it should develop beyond its current existence as an addition to already established schools, theories, and research of the other behavioral sciences (14).

The future of horticultural therapy lies in improving the scientific structure and rigor of research design, as well as improving data reporting methods. For example, sound research that controls or isolates horticultural therapy factors and variables that promote or hinderpatient growth in randomized controlled trialsis needed so that evidence-based practice models can be developed into manualized empirically supported treatments and implemented by practicing horticultural therapists.

\section{Conclusion}

The use of plants and plant-based activities as a therapy-based method will continue to have a role in the healthcare community in countries and cultures that practice horticultural therapy. The practice will undoubtedly expand to other societies as greater recognition of its benefits are achieved. Plants and gardening activity are benign and non-threatening, making “treatments" universally appealing to diverse populations.

\section{References}

1. The American Horticultural Therapy Association. Definitions and positions paper, 2017. https://www.ahta.org/assets/docs/definitions\%20and\%20positio ns\%20final\%206.17.pdf

2. Pandey, D., \& Pandey, V.C. Sacred plants from ancient to modern era: traditional worshiping towards plants conservation. Tropical Plant Research, 2016;136.

3. Davis, S. Development of the profession of horticultural therapy. In Simson, S.P, \& Straus, M.C. (Eds.). Horticulture as therapy: Principles and practice, 1998:3-18. CRC Press.

4. The American Horticultural Therapy Association. Horticultural therapy: History and practice, 2020. https://www.ahta.org/what-is-horticultural-therapy.

5. Relf, D. People-Plant Relationship. In Simson, S.P., \& Straus, M.C. (Eds.). Horticultural as therapy: Principles and practice. 1998:21-42. CRC Press.

6. Haller, R. Vocational, social, and therapeutic programs in horticulture. In Simson, S.P, \& Straus, M.C. (Eds.). Horticulture as therapy: Principles and practice. 1998:43-68. CRC Press.

7. Vita A., \&Barlati, S. The implementation of evidence-based psychiatric rehabilitation: challenges and opportunities for mental health services. Frontiers in Psychiatry. 2019;10. https://doi.org/10.3389/fpsyt.2019.00147

8. HeilmayrD, Reiss Noah \& Buskirk Miranda. Community Gardens Cultivate Positive Experiences for Refugees. Journal of therapeutic Horticulture. 2020;30(1):25-35.

9. Sia A. and Diehl E. Nature-based Activities for Older Adults: A Case Study in Singapore. Journal of therapeutic Horticulture. 2020;30(1):66-75).

10. Matsubayashi K, Kimura S, Iwasaki T, Hamada T, Okumiya K, Fujisawa M, et al. Evaluation of Subjective Happiness in the Elderly Using a Visual Analogue Scale of Happiness in Correlation with Depression Scale. Jpn j geriat.1992;29:811-6. https://doi.org/10.3143/geriatrics.29.811 
11. Kelley RJ, Waliczek TM, Le Duc FA. The Effects of Greenhouse Activities on Psychological Stress, Depression, and Anxiety among University Students Who Served in the U.S. Armed Forces. Horts2017;52:1834-9. https://doi.org/10.21273/hortsci12372-17

12. Toyoda M, Yokota Y, Rodiek S. Gardening May Repeatedly Activate the Frontal Pole. JBBS 2017;07:464-83. https://doi.org/10.4236/jbbs.2017.710033

13. Gladding, S.T. History of, rationale for, and benefits of using the arts in counseling. The creative arts in counseling. 4th ed. Alexandria, VA: American Counseling Association; 2016.

14. Gladding, S.T. Animal-assisted therapy in counseling, therapeutic horticulture, and wilderness/nature therapy. The creative arts in counseling. 4th ed. Alexandria, VA: American Counseling Association; 2016.

15. Gladding ST, editor. The Creative Arts in Counseling. Wiley; 2016. https://doi.org/10.1002/9781119291961

16. Rycroft-Malone J, Seers K, Titchen A, Harvey G, Kitson A, McCormack B. What counts as evidence in evidence-based practice? J Adv Nurs2004;47:81-90. https://doi.org/10.1111/j.1365-2648.2004.03068.x

17. Melnyk, B.M., Finout-Overholt, E., Stillwell, S.B., \& Williamson, K.M. The seven steps of evidence-based practice. The American Journal of Nursing. 2010;110(1). 51-53 